\title{
Oxigenación de músculos respiratorios y locomotores durante el test cardiopulmonar en pacientes con circulación de Fontan: serie de casos
}

\author{
Felipe Contreras-Briceño 1, 2, a; Paulo Valderrama ${ }^{3}$; Eduardo Moya ${ }^{1,}$ a; Maximiliano Espinosa ${ }^{1,}$ a; Yenny Villaseca ${ }^{1,}$ a; Cinthya Ira-Ira ${ }^{3,}$, \\ Anita Moya $^{3, b}$; Jocelyn Mieres ${ }^{3,4}$; Cristián Clavería ${ }^{3}$. \\ 1. Laboratorio de Fisiología del Ejercicio, Departamento Ciencias de la Salud, Facultad de Medicina, Pontificia Universidad Católica de Chile. \\ Avenida Vicuña Mackenna \#4860, Macul, Santiago, Región Metropolitana, Chile. \\ 2. Sección de Fisiología, Departamento de Biología Celular, Fisiología e Inmunología, Facultad de Biología, Universidad de Barcelona. Avenida \\ Diagonal \#643, Barcelona, España. \\ 3. Cardiología Pediátrica, Departamento de Cardiología y Enfermedades Respiratorias Pediátricas, Facultad de Medicina, Pontificia Universidad \\ Católica de Chile. Diagonal Paraguay \#362, Santiago, Región Metropolitana, Chile. \\ 4. Unidad de Cardiología Infantil, Hospital Dr. Sótero del Río. Avenida Concha y Toro \#3459, Santiago, Región Metropolitana, Chile.
}

a Título profesional. Kinesiólogo.

b Título profesional. Enfermera.

Este estudio fue financiado a través del "Premio Investigador Joven 2018 de la Sociedad Chilena de Cardiología", concedido a la Dra. Jocelyn Mieres H.

Antecedentes: Los pacientes con circulación de Fontan (PCF) presentan limitación cardíaca durante el esfuerzo máximo lo que repercute en menor capacidad de ejercicio $\left(\mathrm{VO}_{2}\right.$-peak). La rehabilitación cardiovascular (RC) revierte este desacondicionamiento, al aumentar el gasto cardíaco y diferencia arteriovenosa de oxígeno, aspectos evaluados con monitorización invasiva y gases exhalados. La valoración no invasiva de la saturación muscular de oxígeno $\left(\mathrm{SmO}_{2}\right)$ es un método de reciente aplicación para evaluar la limitación muscular al ejercicio. En PCF esta limitación puede atribuirse a la mayor acción de músculos respiratorios (cambios ventilatorios) y/o locomotores (carga periférica).

Objetivo: Evaluar el trabajo de músculos respiratorios y locomotores durante el ejercicio físico máximo e incremental mediante los cambios en la $\mathrm{SmO}_{2}$.

Métodos: A seis PCF (5 hombres; $13.8 \pm 2.9$ años; $158 \pm 9 \mathrm{~cm} ; 49.8 \pm 13.3 \mathrm{~kg}$ ) se les valoró el $\mathrm{VO}_{2}$-peak $\left(23.0 \pm 4.5 \mathrm{~mL} \cdot \mathrm{kg}^{-1} \cdot \mathrm{min}^{-1}\right)$ mediante ciclo-ergoespirometría sincrónicamente con $\mathrm{SmO}_{2}$ en músculos respiratorios $\left(\mathrm{SmO}_{2}\right.$-m.Intercostales $)$ y locomotores $\left(\mathrm{SmO}_{2}\right.$-m.Vastus-Laterallis) mediante espectroscopía cercana al rango infrarrojo durante el test cardiopulmonar.

Resultados: $\mathrm{SmO}_{2}-$ m.Intercostales disminuyó desde el $60 \%$ del $\mathrm{VO}_{2}$-peak $(\mathrm{p}<0.05)$, mientras que $\mathrm{SmO}_{2}-m$. Vastus-Laterallis no cambió. La ventilación pulmonar (VE) aumentó progresivamente, siendo significativo a partir del $60 \% \mathrm{VO}_{2}$-peak $(\mathrm{p}<0.05)$. La mayor desoxigenación de $\mathrm{SmO}_{2}$-m.Intercostales $\left(\Delta \mathrm{SmO}_{2}\right)$ se asoció con los máximos cambios en ventilación pulmonar $(\Delta \mathrm{V}$ E) en ejercicio (rho= $0.80 ; \mathrm{p}=0.05$ ).

Conclusiones: Durante un protocolo de esfuerzo, los pacientes con circulación de Fontan presentan mayor trabajo muscular respiratorio que locomotor. Los cambios en la ventilación pulmonar se asocian a mayor extracción de oxígeno en la musculatura respiratoria, reforzando la necesidad de incorporar el entrenamiento respiratorio en la rehabilitación cardiovascular. Palabras claves: Ejercicio; Oxigenación muscular; Cardiopatías, Fontan. 


\section{Patients with Fontan: Oxygen Consumption in Respiratory and Locomotor muscles during Excersie Testing.}

Background: During a maximum incremental exercise patients with Fontan circulation (PFC) show cardiac limitation reducing aerobic exercise capacity $\left(\mathrm{VO}_{2}\right.$-peak). Cardiovascular rehabilitation (CR) reverses this deconditioning by increasing cardiac output and arteriovenous oxygen difference, aspects that can be evaluated by invasive methods and analyzing the exhaled gases. Non-invasive assessment of muscle oxygen saturation $\left(\mathrm{SmO}_{2}\right)$ is a novel method for recording local oxygen levels. By this technology, it is possible to evaluate the muscle limitation to exercise. In PFC, that limitation could be attributed to higher contractions of respiratory (ventilatory changes) and/ or locomotor muscles (peripheral load).

Objective: To evaluate in PFC the changes at $\mathrm{SmO}_{2}$ of respiratory and locomotor muscles during a maximum and incremental exercise protocol (cardiopulmonary test, $\mathrm{VO}_{2}$-peak).

Methods: Six PFC (5 men; $13.8 \pm 2.9$ years; $158 \pm 9$ $\mathrm{cm} ; 49.8 \pm 13.3 \mathrm{~kg}$ ) were assessed during the $\mathrm{VO}_{2}-$ peak test $\left(23.0 \pm 4.5 \mathrm{~mL} \cdot \mathrm{kg}^{1}{ }^{1} \mathrm{~min}^{-1}\right)$ by cyclo-ergos- pirometry synchronously with $\mathrm{SmO}_{2}$ at respiratory $\left(\mathrm{SmO}_{2}-\right.$ m.Intercostales $)$ and locomotor $\left(\mathrm{SmO}_{2}-m\right.$. Vastus-Laterallis) muscles by Near-Infrared Spectroscopy (NIRS).

Results: $\mathrm{SmO}_{2}$-m.Intercostales decreased from $60 \%$ of $\mathrm{VO}_{2}$-peak $(\mathrm{p}<0.05)$, while $\mathrm{SmO}_{2}$-m.Vastus-Laterallis did not change. Minute ventilation (VE) increased progressively, showing changes to rest at $60 \%$ of $\mathrm{VO}_{2}$-peak $(\mathrm{p}<0.05)$. The higher deoxygenation of $\mathrm{SmO}_{2}$-m.Intercostales $\left(\Delta \mathrm{SmO}_{2}\right)$ correlated to maximum changes of lung ventilation $(\Delta \mathrm{VE})$ (rho $=0.80 ; \mathrm{p}=0.05$ ).

Conclusions: During an incremental and maximum exercise protocol, patients with Fontan circulation have more work at respiratory than locomotor muscles. Changes in VE are direct associated with greater extraction of oxygen at respiratory muscles, reinforcing the incorporation of respiratory muscle training in cardiovascular rehabilitation.

Keywords: Exercise; Muscle oxygenation; Heart disease, Fontan. 


\section{Introducción:}

La cirugía de Fontan establece una fisiología única mediante la conexión cavopulmonar total, provocando un flujo sanguíneo pasivo tras la ausencia de un ventrículo sub-pulmonar ${ }^{1,2}$. Este procedimiento mejora la supervivencia de los pacientes $^{3}$; sin embargo, la disminución en la capacidad de ejercicio $\left(\mathrm{VO}_{2}\right.$-peak) continúa siendo una de las principales alteraciones en comparación con sujetos sanos ${ }^{4}$. La evidencia reciente sostiene que los pacientes con circulación de Fontan (PCF) con menor $\mathrm{VO}_{2}$ peak presentan mayor riesgo de mortalidad (HR: 0.88; IC 95\%: 0.76-0.98; $\mathrm{p}=0.021^{5}$ ). Durante décadas, se consideró solamente a las variables cardiovasculares (ej. gasto cardíaco, frecuencia cardíaca, volumen expulsivo) como las principales limitaciones para un mejor $\mathrm{VO}_{2}$-peak en PCF, valor cercano al $70 \%$ del alcanzado por niños sanos $^{6,7}$. Otros factores como la función pulmonar restrictiva $(\mathrm{r}=0.86 ; \mathrm{p}<0.001)$, disminuida capacidad de difusión pulmonar $(\mathrm{r}=0.72 ; \mathrm{p}<0.001)^{8}$, debilidad y menor masa muscular periférica $(\mathrm{r}=0.67 ; \mathrm{p}=0.004)^{9}$, también se han asociado con el menor $\mathrm{VO}_{2}$-peak en esta población.

La fisiología univentricular de los PCF y la consecuente menor entrega de flujo sanguíneo (Q) a nivel muscular, implica que durante el ejercicio físico y la mayor actividad contráctil exista incremento en la utilización muscular de oxígeno ${ }^{10}$. Lo anterior provoca una disminución de la saturación de oxígeno a nivel muscular $\left(\mathrm{SmO}_{2}\right)$, lo que puede evaluarse a través de la interpretación de los cambios en las longitudes de onda cercana al rango infrarrojo (Near-Infrared Spectroscopy, NIRS). Así, a través de dispositivos portátiles no invasivos con tecnología NIRS y la cuantificación de la hemoglobina y mioglobina tisular local se determina la $\mathrm{SmO}_{2}$, aspecto que debe ser interpretado como la relación entre el suministro y utilización de oxígeno a nivel de la microcirculación muscular ${ }^{11}$. Utilizando esta tecnología, en PCF se ha evaluado la $\mathrm{SmO}_{2}$ en $m$.Vastus-Laterallis encontrando una disminución total de la oxigenación posterior al ejercicio físico máximo $\left(\Delta \mathrm{SmO}_{2}=4.5 \pm 2.1 \%\right)^{12}$. Hallazgo similar se ha reportado recientemente en $m$.Serratus-Anterior $(\Delta \mathrm{HHb}=$ $48 \pm 5 \%$ ), en donde además se encontró asociación directa con el rendimiento físico $\left(\mathrm{VO}_{2} \text {-peak }\right)^{13}$. Considerando los resultados de estos estudios, se infiere que durante el esfuerzo físico intenso, como ocurre en la valoración del test cardiopulmonar (Cardiopulmonary Exercise Testing, CPET), los PCF presentan incrementos del trabajo muscular locomotor y respiratorio, reflejado en la desoxigenación de $m$.Vastus-Laterallis y $m$.Serratus-Anterior. Sin embargo, y dado las condiciones fisiológicas de los PCF, no se puede aseverar si estos cambios están determinados por el mayor trabajo muscular en sí mismo, o por disminución del flujo sanguíneo y la consecuente entrega de oxígeno local por la mayor acción contráctil de otros grupos musculares exigidos en el esfuerzo físico. Estos cambios se sustentan en las bases fisiológicas del reflejo metabólico ${ }^{14}$. Así, reconocer los cambios en la $\mathrm{SmO}_{2}$ en PCF de los grupos musculares con mayor demanda en la oxigenación durante el ejercicio físico, como son los músculos respiratorios y locomotores, permitiría identificar qué músculos entrenar en los programas de rehabilitación cardiovascular.

El objetivo primario de este estudio fue determinar en PCF el trabajo de músculos respiratorios (m.Intercostales) y locomotores ( $m$.Vastus-Laterallis) mediante el registro de $\mathrm{SmO}_{2}\left(\mathrm{MOXY}{ }^{\circledR}\right)$ durante el ejercicio físico incremental (CPET); y cómo objetivo secundario, evaluar la asociación de $\mathrm{SmO}_{2}$-m.Intercostales con los cambios en las variables ventilatorias.

\section{Métodos:}

En este estudio exploratorio, descriptivo y transversal, se evaluaron seis PCF (5 hombres: $13.8 \pm 2.9$ años; $158 \pm 9$ cm; $49.8 \pm 13.3 \mathrm{~kg}$; IMC: $19.7 \pm 3.9)$. Previo a la selección se realizó un examen físico completo y evaluación de la función pulmonar, con criterios de elegibilidad anteriormente utilizados en la literatura ${ }^{7}$. para realizar CPET $^{15}$. En todos los participantes se evaluó $\mathrm{VO}_{2}$-peak $(23.0 \pm 4.5$ $\mathrm{mL} \cdot \mathrm{kg}^{-1} \cdot \mathrm{min}^{1}{ }^{1}$ ) mediante el análisis de gases exhalados respiración-por-respiración durante el CPET (Corival, Lode, Groningen, Países Bajos), al mismo tiempo que la $\mathrm{SmO}_{2}$ en músculos respiratorios $\left(\mathrm{SmO}_{2}-\right.$ m.Intercostales $)$ y músculos locomotores ( $\mathrm{SmO}_{2}-m$.Vastus-Laterallis) mediante tecnología NIRS. Las evaluaciones se realizaron entre Agosto del 2019 y Enero del 2020 en un centro de referencia de cardiopatías pediátricas de tercer nivel (Servicio de Cardiología Pediátrica, Hospital Clínico Pontificia Universidad Católica de Chile). Este proyecto fue aprobado por el Comité de Ética en Seres Humanos (proyecto número 190121011) considerando la Declaración de Helsinki (1975). Se obtuvo el consentimiento y asentimiento informado por escrito de todos los pacientes y su responsable legal.

\section{Test cardiopulmonar de ejercicio (Cardiopulmonary} Exercise Testing, CPET).

La evaluación de CPET fue realizada por cardiólogo experto a través de la ergoespirometría (Lode Corival CPET, Lode BV, Groningen, Países Bajos) y en bicicleta 


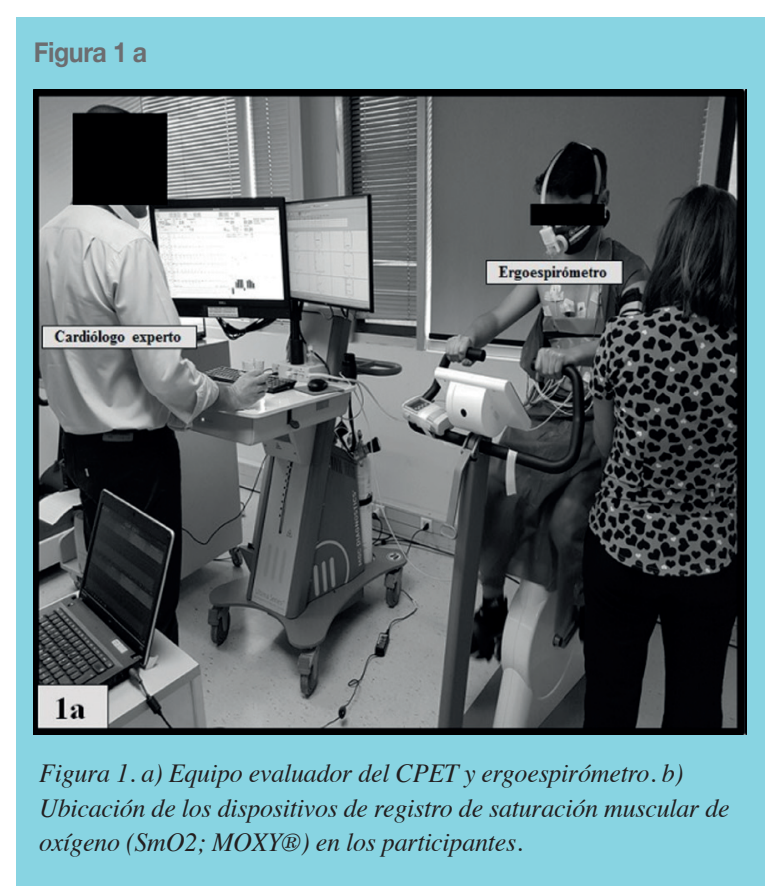

estática (Última CPX, MGC Diagnostics Corporation, Saint Paul, Minnesota, EE.UU) (Figura 1a). El protocolo consistió en una fase inicial de calentamiento de $3 \mathrm{mi}-$ nutos con una carga de 10 vatios, y posteriormente se continuó con la fase de ejercicio utilizando protocolo de medición reportado en la literatura, consistente en $1 \mathrm{mi}$ nuto de aumento incremental de carga (10 o 15 vatios $)^{16}$, obteniendo una duración total del test entre 8-12 minutos. En todo momento se incentivó al paciente a mantener una cadencia de 60 revoluciones por minuto para lograr su máximo esfuerzo. Durante todo el protocolo de ejercicio se evaluó el ritmo cardíaco con un electrocardiograma de 12 derivadas continuo, saturación con oxímetro de pulso, presión arterial (Critikon Dinamap 8100, GE Medical Systems, Milwaukee, EE.UU). Además, se evaluó la percepción subjetiva de esfuerzo mediante escala de Borg modificada ${ }^{17}$. Antes de cada medición, se calibró el analizador de gases siguiendo las instrucciones del fabricante. Las condiciones de temperatura ambiental $\left(20 \pm 1^{\circ} \mathrm{C}\right)$ y humedad relativa $(42 \pm 2 \%)$ del lugar de medición se mantuvieron estable durante todo el protocolo de ejercicio y en cada una de las evaluaciones.

\section{Saturación muscular de oxígeno $\left(\mathrm{SmO}_{2}\right)$}

El registro de $\mathrm{SmO}_{2}$-m.Intercostales y $\mathrm{SmO}_{2}$-m.Vastus-Laterallis fue en forma simultánea durante la prueba de $\mathrm{VO}_{2}$-peak, utilizando dispositivos MOXY® (Moxy, Fortiori Design LLC, Minnesota, EE. UU) el que a través del principio NIRS $(630-850 \mathrm{~nm})$, estima la proporción de hemoglobina y mioglobina unida o no a oxígeno en la microcirculación del territorio muscular, permitiendo la medición de $\mathrm{SmO}_{2}$ en los músculos evaluados como reflejo del trabajo muscular regional. La posición de los dispositivos se realizó mediante un

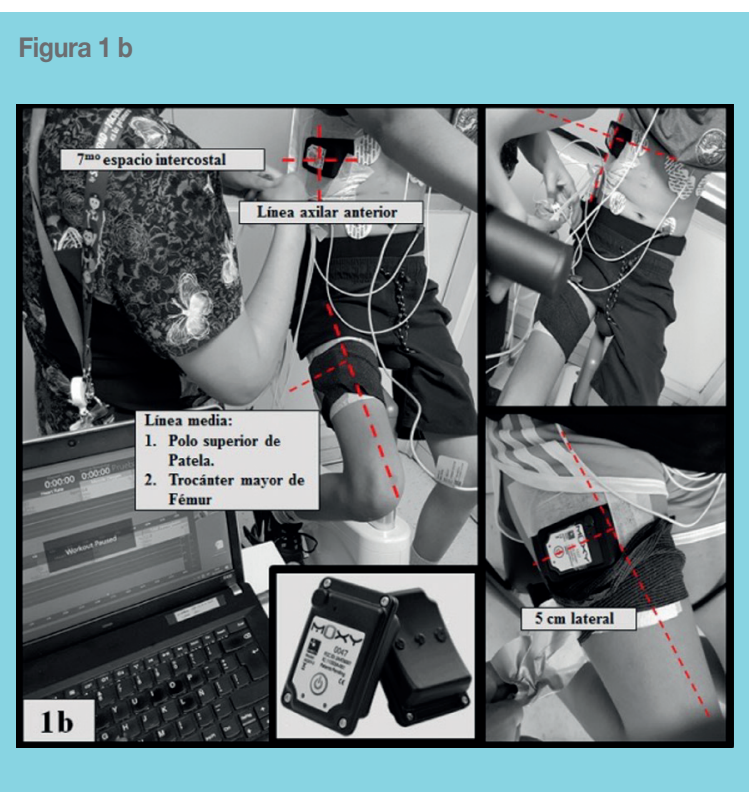

protocolo utilizado previamente por nuestro grupo de investigación (Figura 1b) ${ }^{18}$.

\section{Análisis de datos}

El $\mathrm{VO}_{2}$-peak se calculó como el promedio de los últimos 30 segundos de mayor intensidad de esfuerzo físico durante CPET. Además, la carga durante el ejercicio fue analizada a través del tiempo total desde el inicio hasta el $\mathrm{VO}_{2}$-peak, estimando la carga de trabajo como el porcentaje de $\mathrm{VO}_{2}\left(\%\right.$ de $\left.\mathrm{VO}_{2}\right)$ correspondiente al porcentaje del tiempo durante la prueba desde 0 a $100 \%$, siendo $100 \%$ el tiempo en el que cada participante alcanzó su $\mathrm{VO}_{2}$-peak.

Los valores de $\mathrm{SmO}_{2}$ presentan variabilidad entre grupos musculares principalmente debido a que en su análisis se incluye tanto la cuantificación de hemoglobina como mioglobina (correspondiente alrededor del 30 a $50 \%$ de la señal), cuya concentración es variable dependiendo de las características morfológicas y estructurales de los diferentes grupos musculares. En la literatura científica no existen valores de normalidad como referencia. Con la finalidad de visualizar adecuadamente el comportamiento de las variables ventilatorias analizadas respira- 
Tabla 1. Características clínicas y antropométricas de los pacientes.

\begin{tabular}{|c|c|c|c|c|c|c|c|c|c|}
\hline Paciente & Edad (años) & Sexo & $\operatorname{IMC}\left(\mathbf{k g} / \mathbf{m}^{2}\right)$ & $\begin{array}{c}\text { Ventrículo } \\
\text { predominante }\end{array}$ & Fracción de eyección & Esternotomía (n) & $\begin{array}{c}\text { CF } \\
\text { (NYHA) }\end{array}$ & $\begin{array}{c}\text { Edad Fontan } \\
\text { (meses) }\end{array}$ & Medicamentos \\
\hline 1 & 17 & M & 24,92 & AT-1B & conservada & 3 & 1 & 31 & AA / Enal. \\
\hline 2 & 17 & M & 15,43 & HVI & disminuida & 5 & 2 & 36 & AA / Sild. \\
\hline 3 & 14 & M & 21,97 & $\begin{array}{c}\text { DSVD+AM } \\
\text { DTGA }\end{array}$ & disminuida & 3 & 1 & 28 & AA/Enal. \\
\hline 4 & 11 & M & 15,40 & HVI & conservada & 3 & 1 & 39 & AA/Enal./Esp. \\
\hline 5 & 14 & $\mathrm{~F}$ & 22,46 & HVI & conservada & 3 & 1 & 36 & AA. \\
\hline 6 & 10 & M & 18,09 & HVI & disminuida & 4 & 1 & 33 & AA/ Enal./Sild. \\
\hline
\end{tabular}

Abreviaturas = IMC: Índice de masa corporal; AT-1B: Atresia tricúspide 1B; HVI: Hipoplasia ventrículo izquierdo; DSVD-AM: Doble salida ventrículo derecho con atresia mitral; DTGA: D-Transposición de grandes arterias; CF: Clasificación funcional; NYHA: New York Heart Association; AA: Ácido acetilsalicílico; Enal: Enalapril; Sild: Sildenafil; Esp: Espironolactona.

ción-por-respiración durante CPET, se realizó una estandarización de los valores obtenidos mediante el traspaso a unidades normalizadas o arbitrarias de la ventilación pulmonar (VE (u.a)), volumen corriente (Vc (u.a)) y frecuencia respiratoria ( $\mathrm{Fr}$ (u.a)), considerando el inicio de la prueba como referencia y asignación de valor 1.0. Los cambios obtenidos en el resto de la prueba se expresan proporcionalmente respecto al valor $1.0 \mathrm{y}$ son analizados en los distintos $\%$ de $\mathrm{VO}_{2}$ como el número de veces que incrementan las variables ventilatorias en el ejercicio. Lo anterior, también permite analizar la estrategia ventilatoria utilizada, la cual corresponde a los cambios de Vc o Fr expresados en u.a durante el ejercicio incremental en base al porcentaje de la prueba $\left(\%\right.$ de $\left.\mathrm{VO}_{2}\right)$.

La oxigenación muscular mediante $\mathrm{SmO}_{2}$-m.Intercostales y $\mathrm{SmO}_{2}-m$.Vastus-Laterallis, fueron monitorizados al mismo tiempo desde el inicio de CPET hasta el $\mathrm{VO}_{2}$-peak. Los cambios de $\mathrm{SmO}_{2}$ en ambos grupos musculares se expresaron en intervalos de $10 \%$ (es decir, a $10 \%$ - $\mathrm{VO}_{2}$-peak, $20 \%$ - $\mathrm{VO}_{2}$-peak, $30 \%$ - $\mathrm{VO}_{2}$-peak, etc.), en el que se promedió el registro de $\mathrm{SmO}_{2}$ durante los 30 segundos posteriores al porcentaje de $\mathrm{VO}_{2}$-peak seleccionado.

Además, para visualizar el comportamiento del trabajo respiratorio (Work of Breathing, WOB) durante todo el procedimiento de CPET, se analizó la diferencia de $\mathrm{SmO}_{2}$-m.Intercostales entre la oxigenación inicial y final de la prueba, denominándose como la máxima desoxigenación durante el esfuerzo máximo $\left(\Delta \mathrm{SmO}_{2}\right)$. De igual forma, se analizó la máxima diferencia de VE, corres- pondiente a la resta del valor alcanzado al final de la prueba y el inicio $(\Delta \mathrm{VE})$.

\section{Análisis estadístico}

En consideración al número de participantes evaluados, los datos de $\mathrm{SmO}_{2}$ se presentan como mediana y rango intercuartil. Para determinar las diferencias entre la $\mathrm{SmO}_{2}-m$.Intercostales con la $\mathrm{SmO}_{2}-m$.Vastus-Laterallis y las diferencias de las variables ventilatorias durante el CPET se utilizó el test de Friedman. Para la asociación de $\mathrm{SmO}_{2}$-m.Intercostales con $\mathrm{SmO}_{2}-m$.Vastus-Laterallis y con variables ventilatorias, se utilizó el coeficiente de correlación de Spearman. El nivel de significación estadística se estableció en $\mathrm{p}<0.05$. El software estadístico utilizado fue GraphPad PRISM®) v8.0 (GraphPad Software Inc, California, EE.UU).

\section{Resultados:}

Los datos antropométricos y clínicos de los participantes se presentan en la Tabla 1. Cuatro de los seis pacientes (66.6\%) presentan ventrículo derecho predominante. Todos los PCF presentaban Fontan extracardíaco.

Las variables de función pulmonar y parámetros evaluados durante CPET de los seis participantes se muestran en las Tabla 2 y 3 , respectivamente.

$\mathrm{La} \mathrm{SmO}_{2}-m$.Intercostales disminuyó a partir del $60 \%$ del $\mathrm{VO}_{2}$-peak $(\mathrm{p}<0.05)$, mientras que $\mathrm{SmO}_{2}-m$.Vastus-Laterallis no cambió (Figura 2a). Ambas variables se asociaron directamente $(r h o=0.28 ; p=0.02)$ (Figura 2b). Por otro lado, la VE aumentó durante el protocolo desde el 


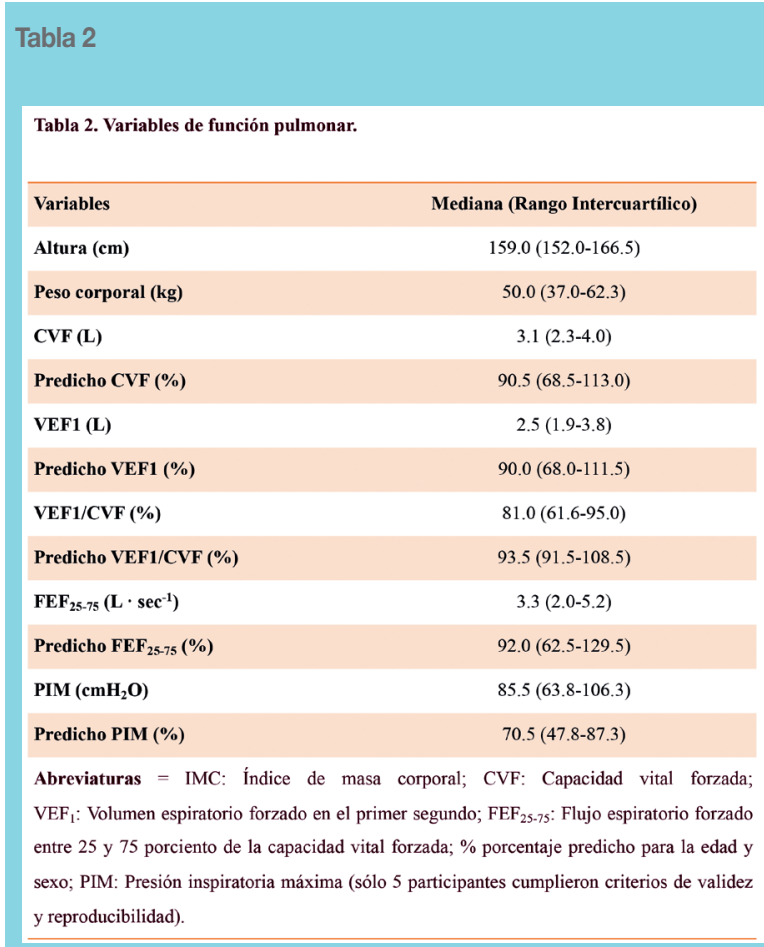

\section{Tabla3}

Tabla 3. Variables del test cardiopulmonar.

\begin{tabular}{|c|c|}
\hline Variables & Mediana (Rango Intercuartílico) \\
\hline $\operatorname{VVM}\left(L \cdot \mathbf{m i n}^{-1}\right)$ & $73.7(57.2-114.2)$ \\
\hline Reserva ventilatoria (\%) & $33.8(31.8-52.1)$ \\
\hline$\dot{\mathrm{V}} \mathrm{E}_{-p e a k}\left(\mathrm{~L} \cdot \mathbf{m i n}^{-1}\right)$ & $47.1(36.5-60.1)$ \\
\hline$\dot{\mathrm{V}} \mathrm{O}_{2 \text {-peak }}\left(\mathrm{ml} \cdot \mathbf{k g}^{-1} \cdot \mathbf{m i n}^{-1}\right)$ & $22.5(15.4-22.8)$ \\
\hline Predicho VंO ${ }_{2-\text { peak }}(\%)$ & $44.3(41.8-71.2)$ \\
\hline$\dot{\mathrm{V}} \mathrm{O}_{2}$ en VT $2\left(\mathrm{ml} \cdot \mathbf{k g}^{-1} \cdot \mathrm{min}^{-1}\right)$ & $12.2(10.2-15.0)$ \\
\hline$\dot{\mathrm{VE}} / \mathrm{VCO}_{2}$ en $\mathrm{VT}_{2}$ & $34.1(33.2-35.2)$ \\
\hline RER peak & $1.18(1.09-1.3)$ \\
\hline Pulso de $\mathrm{O}_{2}\left(\mathrm{~mL} \cdot \mathrm{kg}^{-1} \cdot\right.$ latido $\left.^{-1}\right)$ & $6.6(5.5-8.0)$ \\
\hline $\mathrm{FC}_{\text {-reposo }}\left(\mathrm{L} \cdot \mathrm{min}^{-1}\right)$ & $91(74-104)$ \\
\hline $\mathrm{FC}_{-p e a k}\left(\mathbf{L} \cdot \mathrm{min}^{-1}\right)$ & $170(151-174)$ \\
\hline $\mathrm{SpO}_{2 \text {-reposo }}(\%)$ & $95.5(95.0-96.0)$ \\
\hline $\mathrm{SpO}_{2-p e a k}(\%)$ & $93.5(91.5-94)$ \\
\hline \multicolumn{2}{|c|}{ 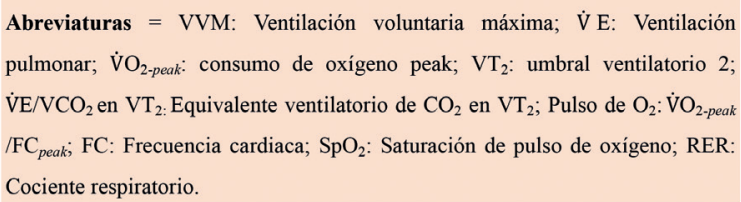 } \\
\hline
\end{tabular}

$60 \%$ del $\mathrm{VO}_{2}$-peak $(\mathrm{p}<0.05)$. La estrategia ventilatoria, muestra que tanto el volumen corriente como la frecuencia respiratoria incrementaron desde $80 \%$ del $\mathrm{VO}_{2}$-peak $(\mathrm{p}<0.05)$ (Figura 2c). La mayor desoxigenación de los m.Intercostales $\left(\Delta \mathrm{SmO}_{2}\right)$ se asoció con la mayor $\mathrm{VE}$ $(\Delta \mathrm{VE})($ rho $=0.80 ; \mathrm{p}=0.05)$ (Figura 2d).

\section{Discusión:}

El propósito de este estudio fue investigar el efecto en los niveles de oxígeno local en dos grupos musculares durante CPET mediante el registro de $\mathrm{SmO}_{2}\left(\mathrm{MOXY}{ }^{\circledR}\right)$ como reflejo del trabajo muscular respiratorio y locomotor en seis niños con circulación de Fontan. Nuestro resultado principal fue que durante el CPET existe una mayor desoxigenación de $\mathrm{SmO}_{2}$-m.Intercostales (músculos respiratorios) que $\mathrm{SmO}_{2}$-m.Vastus Laterralis (músculos locomotores), es decir, aumenta el WOB dado la mayor carga metabólica impuesta por el esfuerzo máximo. Además, el incremento del WOB se asocia fuertemente al cambio en la ventilación pulmonar, lo que indica que a mayor aumento de VE ocurre mayor desoxigenación muscular respiratoria durante el ejercicio, siendo significativo a partir de la carga periférica correspondiente al $60 \%$ del $\mathrm{VO}_{2}$-peak (Figura 2a y $2 \mathrm{~d}$ ).

Con respecto a la oxigenación muscular respiratoria en PCF encontramos resultados similares a Stöcker, et al., quienes evaluaron un grupo de 22 PCF (12 años; IMC: 17.2) durante CPET en cicloergómetro, reportando una mayor desoxigenación muscular respiratoria desde el $40 \%$ al $90 \%$ del $\mathrm{VO}_{2}$-peak, lo que coincidió también con un incremento significativo de la VE en la misma intensidad en comparación a los sujetos sanos del mismo estudio $^{13}$. Asimismo, Moalla, et al. evaluaron a 12 pacientes pediátricos con cardiopatías congénitas encontrando resultados similares en la desoxigenación respiratoria en comparación con sujetos sanos ${ }^{19}$. Por lo tanto, se infiere que el incremento del WOB es una limitación al ejercicio físico en los PCF, lo que se explicaría clínicamente por la mayor disnea manifestada durante grandes esfuerzos. Otro hallazgo interesante en nuestro estudio, fue la no disminución de $\mathrm{SmO}_{2}$-m.Vastus-Laterallis durante el CPET, a pesar del incremento en los vatios o carga periférica (Figura 2a). Si bien se obtuvo una correlación entre la desoxigenación de $\mathrm{SmO}_{2}-m$.Intercostales con $\mathrm{SmO}_{2}-m$. Vastus-Laterallis (Figura 2b), la asociación fue débil (rho $=0.28$ ), y por tanto, es necesario aumentar el número de pacientes evaluados para sostener que el incremento en el WOB es la principal limitación muscular al ejercicio en PCF, y no así el factor muscular periférico ${ }^{13}$. 


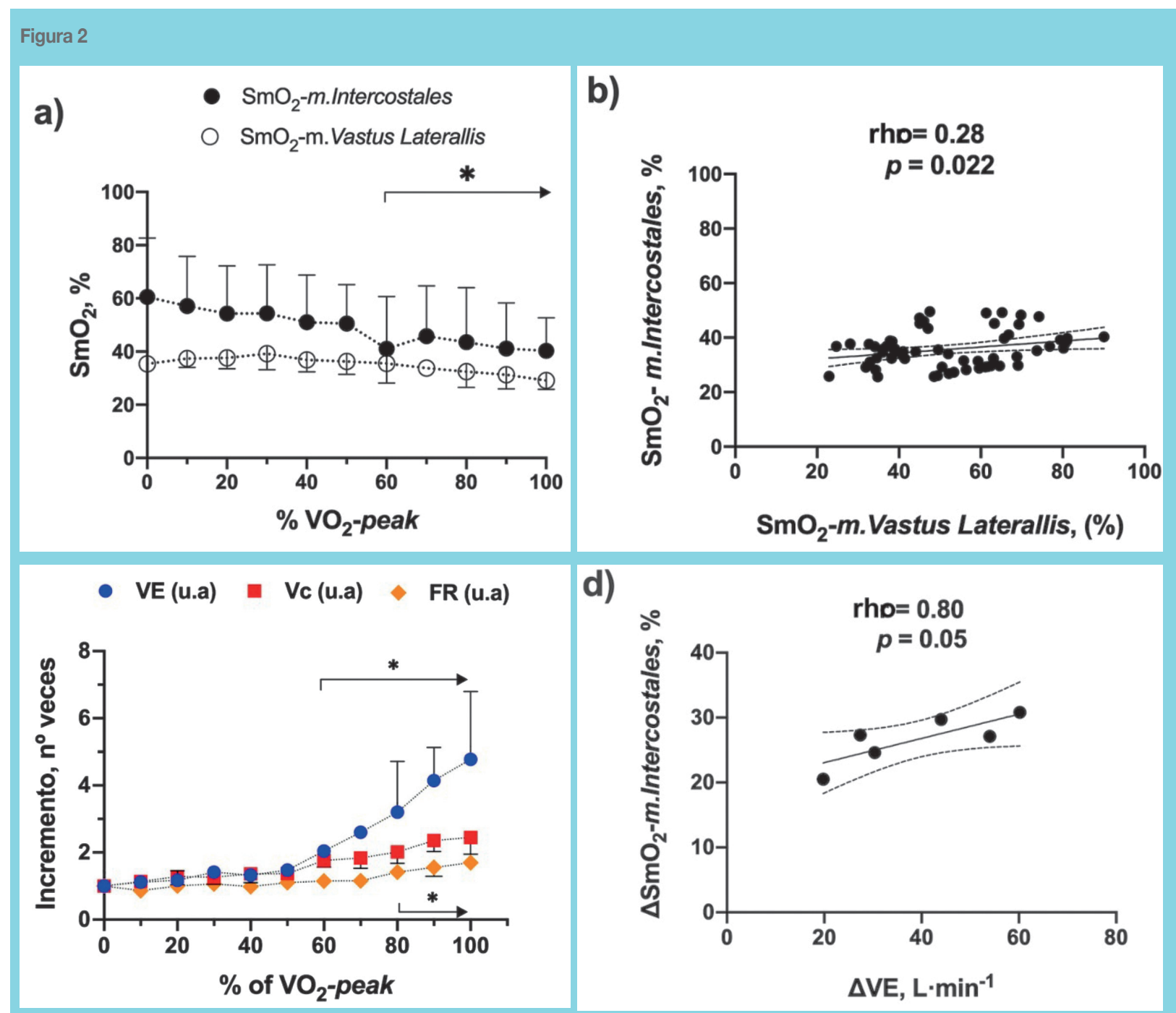

Comportamiento de la oxigenación muscular y variables ventilatorias durante el test cardiopulmonar de seis pacientes con circulación de Fontan. a) Valores absolutos de la saturación muscular de oxígeno (SmO2) de m.Intercostales y m.Vastus-Laterallis según el porcentaje de consumo de oxígeno pico (\% V O2-peak) en intervalos de 10\%.b) Correlación entre valores absolutos de SmO2 de m.Intercostales y m.Vastus-Laterallis. c) Valores normalizados en unidades arbitrarias (u.a) de las variables ventilatorias ventilación pulmonar minuto $(V \cdot E)$, volumen corriente $(V c)$ y frecuencia respiratoria (Fr) según el \% de V O2-peak. d) Correlación de la diferencia de SmO2 de m.Intercostales entre la máxima oxigenación inicial de la prueba y final ( $\triangle \mathrm{SmO2}$ ) con la diferencia entre $V \cdot E$ máxima lograda al final de la prueba y el inicio de $C P E T(\Delta V \cdot E)$. * $=p<0.05$ respecto al inicio de la prueba de esfuerzo cardiopulmonar post test de Friedman.

Escasos son los reportes relacionados a los cambios en la oxigenación de músculos locomotores en niños con cardiopatías. En este sentido, Moalla, et al. evaluó en 9 niños cardiópatas (13.5 años, IMC: 20.3) la oxigenación muscular en condiciones estáticas, en donde se encontró mayor desoxigenación del m.Vastus-Lateralis en altas intensidades de contracción isométrica respecto a sujetos sanos control $\left(\Delta \mathrm{SmO}_{2}: 0.10 \pm 0.03\right.$ u.a, $\left.\mathrm{p}<0.05\right)$; así mismo, encontró menor $\mathrm{SmO}_{2}$ desde el $75 \%$ hasta el $100 \%$ del tiempo total de ejercicio $(5.9 \pm 1.4$ y $8.5 \pm 2,4$ u.a, respectivamente), lo que se asoció a menor fuerza y resisten- cia muscular ${ }^{20}$. Por otro lado, Dandurán, et al., evaluó en 50 PCF (15.4 años) la $\mathrm{SmO}_{2}-m$.Vastus-Lateralis en cinta rodante durante un test físico incremental, reportando diferencias significativas respecto a sujetos sanos $\left(\Delta \mathrm{SmO}_{2}\right.$ : 17\%, rango intercuartil: 12-18), lo que se acentuó más en el tiempo de recuperación (5-minutos posteriores), y no así en reposo $^{21}$. Sin embargo, ambos autores señalan que existe un menor rendimiento muscular durante el ejercicio en PCF, al compararlo con niños sanos dado una menor saturación regional y un menor flujo sanguíneo durante el ejercicio y posterior recuperación. 
Los resultados de nuestro estudio son similares a lo reportado por Vandekerckhove, et al., quienes encontraron en 18 PCF (11.8 años; IMC 18.3) mayor desoxigenación del $m$.Vastus-Lateralis que los controles sanos desde los 0 a 70 vatios durante la valoración de CPET; sin embargo, los niños sanos lograron mayor desoxigenación $(56.0 \pm 3.9 \%)$ que los PCF $(59.5 \pm 2.1 \%)$ al término de la prueba. Además, los PCF presentaron un comportamiento de desoxigenación constante no significativo durante toda la prueba y no alcanzaron un nivel esperable de extracción máxima de oxígeno (meseta) $(3.57 \pm 1.22 \mu \mathrm{mol})$ como si lo hicieron los controles $(5.07 \pm 2.10 \mu \mathrm{mol})^{12}$. Lo anterior, es concordante con nuestros hallazgos, lo que podría indicar que la musculatura periférica no alcanza una máxima capacidad de desoxigenación durante el ejercicio en esta población. Esto se puede atribuir a alteraciones periféricas que presentan los PCF, tales como atrofia muscular con cambios en el tipo de fibras ${ }^{9,22}$, disfunción endotelial y autonómica con aumento de la resistencia vascular general ${ }^{23}$, limitación del flujo sanguíneo esquelético $^{24}$ y/o miopenia asociada a la circulación de Fontan $^{25}$, lo que podría explicar la limitada desoxigenación periférica.

Otro hallazgo interesante fue la estrategia ventilatoria utilizada en esta población para incrementar la VE, la que se hace notoria a intensidades por sobre el $80 \%$ del $\mathrm{VO}_{2-}$ peak, al aumentar tanto la Fr como el Vc (Figura 2c).

Al contrario de estos pacientes, la estrategia ventilatoria de sujetos sanos con fisiología biventricular durante CPET a altas intensidades es el aumento de la VE principalmente por incrementos exponenciales de la $\mathrm{Fr}^{26}$. No obstante, la estrategia utilizada por los participantes de este estudio podría explicarse como un mecanismo compensatorio tras la ausencia de un ventrículo sub-pulmonar que, a través de la contracción de la musculatura respiratoria provocaría mayores gradientes de presión pleural negativa a expensas de incrementos en Vc durante el ejercicio, elevando el llenado univentricular para optimizar la entrega de sangre a los tejidos en cada respiración ${ }^{27}$.

En los últimos años ha existido creciente evidencia sobre la evaluación de NIRS en pacientes pediátricos con cardiopatías congénitas 12,28 . Según nuestro conocimiento, este es el primer reporte que evalúa en PCF la oxigenación muscular respiratoria y locomotora de manera sincrónica en población chilena, entregando resultados interesantes respecto a la necesidad de valorar la oxigenación muscular respiratoria y locomotora como un componente objetivo para la prescripción individualizada en la reha- bilitación cardíaca sugerida para los $\mathrm{PCF}^{6,22}$.

En concordancia con nuestros resultados, sugerimos la incorporación en forma rutinaria del entrenamiento muscular respiratorio dentro de las programas de rehabilitación cardiovascular, tal como se ha sido sugerido en otros estudios mediante intervenciones en la musculatura respiratoria para esta población a través de un sustento fisiológico, pero sin una valoración del WOB que refleje la necesidad del entrenamiento específico para este grupo muscular 29,30.

Existen algunas limitaciones en este estudio como el reducido número de participantes evaluados, y la no incorporación de un grupo control, por lo que los datos deben ser interpretados con precaución. Además, no se evaluó el grosor del tejido adiposo subcutáneo en los sitios que se instalaron los dispositivos MOXY $\AA$, tal como es sugerido en la literatura ${ }^{11}$. Sin embargo, en este estudio los pacientes eran delgados y presentaban un IMC cercano al límite inferior de peso normal, por lo que se infiere la no presencia de artefactos durante nuestras mediciones. A futuro es necesario realizar estudios clínicos controlados y randomizados en los que se evalúen los efectos de programas de entrenamiento físico y respiratorio sobre los cambios en los niveles de oxigenación muscular local (tanto en músculos respiratorios como locomotores) durante el test cardiopulmonar, con la finalidad de determinar la limitación muscular al esfuerzo físico en PCF.

\section{Conclusión:}

En un protocolo de esfuerzo máximo, los pacientes con circulación de Fontan muestran más trabajo muscular respiratorio que locomotor, evaluado a través del registro de $\mathrm{SmO}_{2}$, lo que podría explicar en parte, la gran disnea referida a esfuerzos físicos de alta intensidad. Los cambios en la ventilación pulmonar se asocian a los niveles de oxígeno en m.Intercostales, lo que refuerza la incorporación del entrenamiento respiratorio en la rehabilitación cardiovascular. El trabajo de extremidades inferiores no sería una limitante para el logro del $\mathrm{VO}_{2}$-peak en esta población, dado las posibles alteraciones propias de la enfermedad en la musculatura periférica.

\section{Agradecimientos:}

A todos los participantes y cuidadores legales por participar activamente y en forma desinteresada en las evaluaciones realizadas.

\section{Conflicto de Interés.}

Los autores declaran no tener conflicto de interés. 


\section{Referencias}

1. GEWILLIG M, BROWN S. The Fontan circulation after 45 years: Update in physiology. Heart. 2016; 102:1081-1086.

2. GEWILLIG M. The Fontan circulation. Heart. 2005; 91:839846.

3. TWEDDELL J, NERSESIAN M, MUSSATTO K, NUGENT M, SIMPSON P, MITCHELL M, et al. Fontan Palliation in the Modern Era: Factors Impacting Mortality and Morbidity. Ann Thorac Surg. 2009; 88:1291-1299.

4. HOCK J, REINER B, NEIDENBACH R, OBERHOFFER R, HAGER A, EWERT P, et al. Functional outcome in contemporary children with total cavopulmonary connection - Health-related physical fitness, exercise capacity and health-related quality of life. Int J Cardiol. 2018; 255:50-54.

5. OHUCHI H, NEGISHI J, NORITAKE K, HAYAMA Y, SAKAGUCHI H, MIYAZAKI A, et al. Prognostic value of exercise variables in 335 patients after the Fontan operation: A 23-year single-center experience of cardiopulmonary exercise testing. Congenit Heart Dis. 2015; 10:105-116.

6. SUTHERLAND N, JONES B, D'UDEKEM Y. Should We Recommend Exercise after the Fontan Procedure? Hear Lung Circ. 2015; 24:753-768.

7. PARIDON S, MITCHELL P, COLAN S, WILLIAMS R, BLAUFOX A, LI J, et al. A Cross-Sectional Study of Exercise Performance During the First 2 Decades of Life After the Fontan Operation. J Am Coll Cardiol. 2008; 52:99-107.

8. TURQUETTO A, CANÊO L, AGOSTINHO D, OLIVEIRA P, LOPES M, TREVIZAN P, et al. Impaired Pulmonary Function is an Additional Potential Mechanism for the Reduction of Functional Capacity in Clinically Stable Fontan Patients. Pediatr Cardiol. 2017; 38:981-990.

9. CORDINA R, O'MEAGHER S, GOULD H, RAE C, KEMP G, PASCO J, et al. Skeletal muscle abnormalities and exercise capacity in adults with a Fontan circulation. Heart. 2013; 99:1530-1534.

10. LA GERCHE A, GEWILLIG M. What Limits Cardiac Performance during Exercise in Normal Subjects and in Healthy Fontan Patients?. Int J Pediatr. 2010; 2010:1-8.

11. TANAKA T, BASOUDAN N, MELO L, WICKERSON L, BROCHARD L, GOLIGHER E, et al. Deoxygenation of inspiratory muscles during cycling, hyperpnoea and loaded breathing in health and disease: a systematic review. Clin Physiol Funct Imaging. 2018; 38:554-565.
12. VANDEKERCKHOVE K, COOMANS I, MOERMAN A, PANZER J, DE GROOTE K, DE WILDE H, et al. Differences in cerebral and muscle oxygenation patterns during exercise in children with univentricular heart after Fontan operation compared to healthy peers. Int J Cardiol. 2019; 290:86-92.

13. STÖCKER F, NEIDENBACH R, FRITZ C, OBERHOFFER R, EWERT P, HAGER A, et al. Oxygen availability in respiratory muscles during exercise in children following Fontan operation. Front Pediatr. 2019; 7:1-8.

14. BRASSARD P, POIRIER P, MARTIN J, NOËL M, NADREAU E, HOUDE C, et al. Impact of exercise training on muscle function and ergoreflex in Fontan patients: A pilot study. Int J Cardiol. 2006; 107:85-94.

15. MCCRINDLE B, ROTH S, SAUL J. Facilitate Future Clinical Trials in Children With the. 2014; 152:427-433.

16. AMEDRO P, GAVOTTO A, GUILLAUMONT S, BERTET H, VINCENTI M, DE LA VILLEON G, et al. Cardiopulmonary fitness in children with congenital heart diseases versus healthy children. Heart. 2018; 104:1026-1036.

17. ROBERTSOU RJ, GOSS FL. Validation O F the Omni Scale of Perceived Exertion. 2008; 1:181-188.

18. CONTRERAS F, ESPINOSA M, HEVIA G, LLAMBIAS D, CARRASCO M, CERDA F, et al. Reliability of NIRS portable device for measuring intercostal muscles oxygenation during exercise. J Sports Sci. 2019; 37:2653-2659.

19. MOALLA W, DUPONT G, TEMFEMO A, MAINGOURD Y, WESTON M, AHMAIDI S. Assessment of exercise capacity and respiratory muscle oxygenation in healthy children and children with congenital heart diseases. Appl Physiol Nutr Metab. 2008; 33:434-440.

20. MOALLA W, DUPONT G, COSTES F, GAUTHIER R, MAINGOURD Y, AHMAIDI S. Performance and muscle oxygenation during isometric exercise and recovery in children with congenital heart diseases. Int J Sports Med. 2006; $27: 864-869$

21. DANDURAN M, DIXON J, RAO R. Near infrared spectroscopy describes physiologic payback associated with excess postexercise oxygen consumption in healthy controls and children with complex congenital heart disease. Pediatr Cardiol. 2012; 33:95-102.

22. CORDINA R, O'MEAGHER S, KARMALI A, RAE C, LIESS C, KEMP G, et al. Resistance training improves car- 
diac output, exercise capacity and tolerance to positive airway pressure in Fontan physiology. Int J Cardiol. 2013; 168:780788.

23. LAMBERT E, D'UDEKEM Y, CHEUNG M, SARI CI, INMAN J, AHIMASTOS A, et al. Skeletal muscle hemodynamics and endothelial function in patients after Fontan operation. Int J Cardiol. 2013; 93:792-797.

24. ROSS F, ARAKAKI L, CIESIELSKI W, MCMULLAN D, RICHARDS M, GEIDUSCHEK J, et al. Assessment of muscle oxygenation in children with congenital heart disease. Paediatr Anaesth. 2019; 29:850-857.

25. TRAN D, AMBROSIO P, VERRALL C, ATTARD C, BRIODY J, SOUZA M, et al. Body Composition in Young Adults Living With a Fontan Circulation: The Myopenic Profile. J Am Heart Assoc. 2020; 9:1-10.

26. SHEEL A, ROMER L. Ventilation and Respiratory Mechanics - Comprehensive Physiology. Compr Physiol. 2012; 2:1093-1142.
27. VAN DE BRUAENE A, CLAESSEN G, LA GERCHE A, KUNG E, MARSDEN A, DE MEESTER P, et al. Effect of respiration on cardiac filling at rest and during exercise in Fontan patients: A clinical and computational modeling study. IJC Hear Vasc. 2015; 9:100-108.

28. VANDEKERCKHOVE K, PANZER J, COOMANS I, MOERMAN A, DE GROOTE K, DE WILDE H, et al. Different Patterns of Cerebral and Muscular Tissue Oxygenation 10 Years After Coarctation Repair. Front Physiol. 2019; 10:1-10.

29. LAOHACHAI K, WINLAW D, SELVADURAI H, GNANAPPA G, D'UDEKEM Y, CELERMAJER D, et al. Inspiratory muscle training is associated with improved inspiratory muscle strength, resting cardiac output, and the ventilatory efficiency of exercise in patients with a Fontan circulation. J Am Heart Assoc. 2017; 6(8):1-11.

30. AIT ALI L, PINGITORE A, PIAGGI P, BRUCINI F, PASSERA M, MAROTTA M, et al. Respiratory Training Late After Fontan Intervention: Impact on Cardiorespiratory Performance. Pediatr Cardiol. 2018; 39:695-704. 